\title{
Comparison of content and psychometric properties for assessment tools used for brain tumor patients: a scoping review
}

Lelde G̦iga' ${ }^{1}$, Anete Pētersone ${ }^{1,2}$, Silva Čakstiña ${ }^{1}$ and Guna Bērzin,a $a^{1,2,3^{*}}$ (i)

\begin{abstract}
Aims: To determine the most frequently utilized functional status assessment instruments for patients with brain tumors, compare their contents, using the International Classification of Functioning, Disability and Health (ICF), and their psychometric properties.
\end{abstract}

Methods: A scoping review was conducted to explore possible assessment instruments and summarize the evidence. A systematic literature search was performed for identification of the frequently used functional assessment tool in clinical trials in PubMed, ScienceDirect, and ProQuest databases. The content of most used instruments was linked to the ICF categories. The psychometric qualities of these assessment tools were systematically searched and analyzed.

Results: Nine most used assessment tools in clinical trials were identified. The most frequently used assessment instrument is the Karnofsky Performance Scale, which is developed for a general assessment of oncological patients. Out of four self-assessment tools, two were disease-specific (EORTC QLQ-BN20 and FACT-Br), EORTC QLQ-C30 has been shown good psychometric properties in patients with brain tumors as well as in patients with various oncological diseases, similar to the SF-36, it is used in patients with brain tumors as well as in patients with various diseases. The Functional Independence Measure and the Barthel Index were two objective assessment tools that described functioning, but two were neuropsychological tests (MMSE and Trial Making Test). Two hundred eighty-three meaningful concepts were identified and linked to 102 most relevant second-level categories covering all components of the ICF. Forty-nine studies reporting psychometric properties of those nine assessment tools were identified, indicating good reliability and validity for all the instruments.

Conclusion: Nine most frequently utilized functional status assessment instruments for patients with brain tumors represent all components of the ICF and have good psychometric properties. However, the choice of the tool depends on the clinical question posed and the aim of its use.

Keywords: International Classification of Functioning, Disability and Health (ICF), Linking, Psychometric properties, Outcome measures, Brain Tumor

*Correspondence: berzina.guna@gmail.com

${ }^{1}$ Riga Stradinš University, Riga, Latvia

Full list of author information is available at the end of the article

\section{Introduction}

Based on 2015 statistics, patients with brain tumors make up a total of $5 \%$ of all oncology patients in Latvia [1]. As the medical industry, diagnostic capabilities, and technologies for treating primary tumors evolve, the survival rates for individuals diagnosed with primary brain tumors have increased significantly $[1,2]$. original author(s) and the source, provide a link to the Creative Commons licence, and indicate if changes were made. The images or other third party material in this article are included in the article's Creative Commons licence, unless indicated otherwise in a credit line to the material. If material is not included in the article's Creative Commons licence and your intended use is not permitted by statutory regulation or exceeds the permitted use, you will need to obtain permission directly from the copyright holder. To view a copy of this licence, visit http://creativecommons.org/licenses/by/4.0/. The Creative Commons Public Domain Dedication waiver (http://creativeco mmons.org/publicdomain/zero/1.0/) applies to the data made available in this article, unless otherwise stated in a credit line to the data. 
Tumor localization, anatomical distribution, and volume are determinants before and after primary treatment. The most common symptoms for brain tumors usually include headache, nausea, vomiting, partial and generalized seizures, cognitive impairment, and ataxia. These symptoms may also arise from common treatment strategies used for brain tumor patients such as chemotherapy, radiation therapy, and surgery. It is estimated that $75 \%$ of all patients with brain tumors show symptoms of focal neurological deficiency [3], which greatly affects one's level of functioning, as well as the quality of life.

Numerous articles discuss the role of rehabilitation in tumor cases, while others discuss the positive effects of rehabilitation for patients with brain tumors compared to patients with stroke or after a traumatic brain injury [4, 5]. All of these articles demonstrate positive outcomes in restoring functioning [6, 7]. Bartolo M. et al. have demonstrated that rehabilitation is very effective if initiated as early as possible after primary treatment for brain tumor patients [5].

To assess the rehabilitation needs and outcomes for this population, a specific functional disability assessment tool is necessary [8]. The use of appropriate assessment tools could improve rehabilitation planning that in turn would lead to better outcomes, including patients' quality of life. Currently, no standardized protocols are provided for evaluation of persons with brain tumors. The International Classification of Functioning, Disability and Health (ICF) provides a framework for coding large-scale health information, a common standardized language for identifying and comparing functional assessment tools, and provides valuable information to develop an evidencebased standardized evaluation protocol for patients with brain tumor [9].

The aim of this study was to determine the most frequently utilized functional assessment instruments for patients with brain tumors, compare their contents, using the International Classification of Functioning, Disability and Health, and analyze their psychometric properties.

\section{Methods}

\section{Identification of assessment tools}

A scoping review was conducted according to Joanna Briggs institute guidelines [10]. PubMed, ScienceDirect, and ProQuest databases were searched (last updated August 2020) for publications since 2000 using the following keywords: "brain neoplasm" or "meningioma" or "glioblastoma" or "intracranial neoplasm" or "brain cancer" or "outcome assessment" or "treatment outcome" or functional outcome mentioned in the title/abstract. Studies referring in the title or abstract to assessment tools used to assess people diagnosed with brain tumors older than 18 years were included. Original research studies randomized controlled clinical trials, observational studies, cross-sectional studies, qualitative studies were included in which authors reported using a tool to assess functioning in persons with brain tumors. Studies were included even if the tool was not initially designed to assess functioning. Studies were excluded if they addressed genetic, laboratory, and animal research. Systematic reviews, secondary analyses of published data, validity studies, protocols, letters, were also excluded from this report. All searches were limited to journal articles written in English; the search results were compiled in the reference management system EndNote where duplicates were removed. A summary of the search procedure is shown in Fig. 1.

Data collection was based on the Joanna Briggs Institute Manual for Evidence Synthesis Chapter 11.2 Development of a Scoping Review protocol [10]. General study data (year of publication, country, study design), available data on participants (number, diagnosis), and assessment tools used in the study were recorded. Assessment tools that were used in more than $9 \%$ of all studies the scoping review using frequency analysis. The choice of this cut-off point was based on the distribution of frequencies as well as on substantive considerations of the list of instruments.

\section{Linking to the ICF}

All assessment instruments identified in the study meeting selection criteria were classified using the ICF linking guidelines. The ICF linking guidelines state that before starting the process of linking health-status measures to the ICF categories, identification of all meaningful concepts within each item of the health status measure needs to be performed. According to the rules, the interval of time cannot be linked to the ICF, also, if a meaningful concept of an item is explained by examples, both the concept and the examples are to be linked, while technical measures can be linked by defining the purpose and then linking it with the ICF category $[11,12]$. Two independent medical professionals (authors LG and SS) separately identified the meaningful concepts within the analyzed instruments and linked them to the ICF concepts. The raters met and discussed any discrepancies to achieve a consensus classification for the instruments and GB served as a third rater, in case the consensus could not be reached. Identified categories within each of the analyzed instruments were organized according to the structure of the ICF. Further, the content of the instruments was compared to identify categories that overlap between the instruments and those that are unique for specific tools. The perspective adopted in health information and categorization of response for self-assessment tools were also reported [11]. 


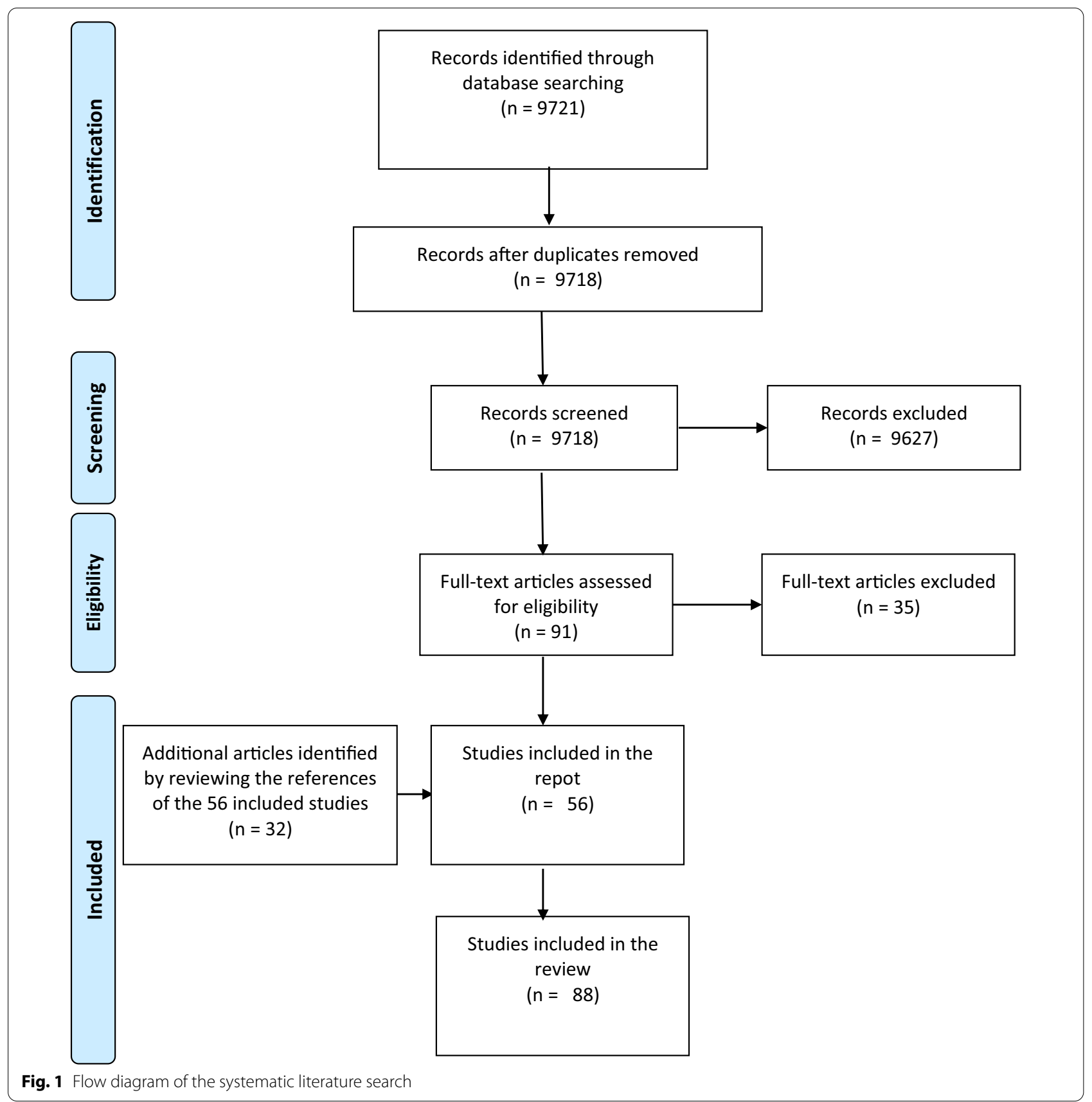

\section{Psychometric properties}

Following the search methodology developed by PubMed [13], the electronic database MEDLINE (PubMed) was searched for studies that reflect the psychometric properties of a particular assessment tool. First, a search was performed using a diagnosis-specific $\mathrm{MeSH}$ terms and key words identified in the search methodology and the names of assessment tools. Headline screening identified studies that reflected one of the psychometric properties of a given instrument (reliability: internal coherence; test/retest method, evaluator reliability. Validity: content validity; criterion validity; construct validity) specific to patients with brain tumors. If psychometric properties for chosen assessment tools were not identified, the search was repeated excluding diagnosis-specific $\mathrm{MeSH}$ terms, thus conducting a search for studies covering different diagnoses. Headline screening then identified studies that reflected one of the psychometric properties of a given instrument for various diagnoses. The interpretation of the psychometric properties is given in Table 1. 
Table 1 The interpretation of the psychometric properties

\begin{tabular}{|c|c|c|}
\hline \multicolumn{3}{|l|}{ Reliability } \\
\hline \multirow[t]{2}{*}{ Internal reliability } & + & Cronbach's a or ICC $\geq 0.70$ \\
\hline & - & Cronbach's a or ICC $<0.70$ \\
\hline \multirow[t]{2}{*}{ Test/retest method } & + & ICC $\geq 0.70$ or Pearson correlation coefficient/ Spearman rank correlation coefficient $\geq 0.80$ \\
\hline & & ICC $<0.70$ or Pearson correlation coefficient/Spearman rank correlation coefficient $<0.80$ \\
\hline \multirow[t]{2}{*}{ Interrater reliability } & & $I C C \geq 0.70$ or Pearson correlation coefficient/ Spearman rank correlation coefficient $\geq 0.80$ \\
\hline & & ICC $<0.70$ or Pearson correlation coefficient/Spearman rank correlation coefficient $<0.80$ \\
\hline \multicolumn{3}{|l|}{ Validity } \\
\hline \multirow[t]{2}{*}{ Content validity } & + & $\begin{array}{l}\text { The content of the assessment instrument is adequate, comprehensive, questions and tasks chosen to adequately reflect the } \\
\text { content to be evaluated }\end{array}$ \\
\hline & - & Not all selected questions and tasks reflect the content, content is not relevant, comprehensive \\
\hline \multirow[t]{2}{*}{ Criterion validity } & + & Significant and stable relation between measurement and another instrument $(r \geq 0.70)$ or with start/end measurement \\
\hline & - & Poor measurement correlation with another instrument $(r<0.70)$ or start / end measurement \\
\hline \multirow[t]{2}{*}{ Structural validity } & + & $\begin{array}{l}\text { Correlation with instruments measuring the same } \geq 0.50 \text { or correlation higher for unrelated elements in the instrument than } \\
\text { for unrelated ones }\end{array}$ \\
\hline & & $\begin{array}{l}\text { Correlation with instruments measuring the same }<0.50 \text { or correlation with related elements in the instrument is lower than } \\
\text { unrelated ones }\end{array}$ \\
\hline \multicolumn{3}{|l|}{ Responsiveness } \\
\hline \multirow[t]{2}{*}{ Responsiveness } & & Able to detect clinically significant changes over time \\
\hline & & Cannot detect clinically significant changes over time \\
\hline
\end{tabular}

Cronbach's a, Chronbach's a coefficient; ICC, interclass correlation coefficient

\section{Results}

\section{Identification of assessment tools}

The initial search strategy returned 9721 articles. The duplicates were removed, titles and summaries were revised, following the exclusion of articles that did not meet the selection criteria, in result 56 articles were included in the scoping review.

To make the search as comprehensive as possible, references from the 56 included articles were studied and an additional 32 articles were included after applying the selection criteria.

As a result, a total of 88 studies were included in the report; 31 were administered in the United States, 42 in Europe (8 in Italy, 8 in the Netherlands, 6 in Norway, 4 in France, Germany and England each 3, Austria, Turkey, Sweden 2 studies each, Poland, Switzerland, Denmark, and Finland each 1), 2 in Australia, 7 in Canada, and 4 in Asian countries (Korea, Israel, Iran). The studies look at groups of patients with various brain tumor diagnoses. The 74 articles included patients with primary tumors, of which 26 were diagnosed with glioma, 3- oligodendroglioma, 1- oligoastrocytoma, 3- astrocytoma, 4- adenoma, 1- meningioma, 1 case study had a mixed group with patients suffering from meningioma and glioblastomas. 28 of the studies did not categorize patients by their histologic type; instead, patients with primary brain tumors were evaluated. 9 studies evaluated patients with secondary brain tumors or with brain metastases. In 4 of the included studies, the functional abilities of patients with brain tumors are compared to those of a stroke patient or a patient with a brain injury.

All instruments mentioned in the articles were identified, yielding 86 assessment tools which are summarized in Additional file 1. According to research methodology, 9 assessment tools that were used in more than $9 \%$ of the research articles included in the study were used for further analysis: A list of these instruments, their abbreviations, and the number of articles that have used that instrument are summarized in Table 2. Out of nine instruments included in the study,

two are specific for patients with brain tumors: ORTC Quality of Life Questionnaire-Brain Neoplasm 20 (EORTC QLQ-BN20), Functional Assessment of Cancer Therapy-Brain (FACT-Br), one is specific for patients with oncological diseases-Karnofsky Performance Scale (KPS), four: Mini-mental State Examination (MMSE), Functional Independence Measure (FIM), Trial Making Test (TMT), Barthel Index (BI) and 36-Item Short Form Health Survey (SF-36)) are used for patients with various diagnoses. The EORTC Quality of Life Questionnaire 30 (EORTC QLQ-C30) has been shown to be valid, reliable, and responsive in patients with brain tumors as well as in patients with various oncological diseases. Similar, to the 36-item Short Form Health Survey (SF-36), it is used in patients with brain tumors as well as in patients with various diseases. 
Table 2 The most frequently used assessment instruments

\begin{tabular}{lllc}
\hline Assessment instrument & Abbreviation & N of studies mentioned & Frequency (\%) \\
\hline Karnofsky Performance Scale & KPS & 42 & 48 \\
Mini-Mental State Examination & MMSE & 20 & 23 \\
EORTC Quality of Life Questionnaire-Core 30 & EORTC QLQ-C30 & 18 & 20 \\
EORTC Quality of Life Questionnaire-Brain Neoplasm 20 & EORTC QLQ-BN20 & 15 & 17 \\
Functional Independence Measure & FIM & 13 & 13 \\
Trail Making Test & TMT & 9 & 15 \\
Barthel Index & BI & 8 & 10 \\
Functional Assessment of Cancer Therapy-Brain & FACT-BR & 8 & 9 \\
36-Item Short Form Health Survey & SF-36 & 9 \\
\hline
\end{tabular}

\section{Linking to the ICF}

In total, 283 meaningful concepts were identified within all nine assessment instruments and linked to 394 most precise categories of the ICF. The detailed description of the linking is shown in Table 3. In two cases, the meaningful concepts could be linked most precisely to the component of Activities and Participation. In 12 cases, it was the first level or chapter under the component of Activities and Participation. The content of the assessment tools was linked to 102 most relevant second-level categories of the ICF in total. Thirty-four of these categories were under the component of Body Functions and

Table 3 Summary of linking the nine most frequently used assessment tools to the ICF

\begin{tabular}{|c|c|c|c|c|c|c|c|c|c|}
\hline & KPS & MMSE & EORTC QLQ-C30 & $\begin{array}{l}\text { EORTC } \\
\text { QLQ-BN20 }\end{array}$ & FIM & TMT & $\mathrm{BI}$ & FACT-BR & SF-36 \\
\hline $\mathrm{N}$ of meaningful concepts & 32 & 11 & 42 & 22 & 19 & NA & 32 & 63 & 62 \\
\hline $\mathrm{N}$ of categories identified & 71 & 18 & 52 & 29 & 31 & 16 & 39 & 66 & 72 \\
\hline $\mathrm{N}$ of unique categories identified & 22 & 15 & 39 & 19 & 29 & 16 & 22 & 52 & 27 \\
\hline Perspective & Desc & Desc & Appr & Appr & Dep & Desc & Dep & Appr & Appr \\
\hline Categorization & & & Int & Int & & & & Int & Int \\
\hline \multicolumn{10}{|l|}{ Body functions } \\
\hline 2nd level & & 4 & 9 & 7 & 3 & 6 & 2 & 16 & 4 \\
\hline $3 \mathrm{~d}$ and 4 th level & & 10 & 6 & 6 & & 1 & & 5 & \\
\hline \multicolumn{10}{|l|}{ Body structures } \\
\hline 2nd level & & & & & & & & 2 & \\
\hline Activities and participation & 1 & & & & & & & & 1 \\
\hline 1st level & 1 & & 5 & & & & & 2 & 4 \\
\hline 2nd level & 4 & & 15 & 5 & 20 & 9 & 8 & 17 & 6 \\
\hline 3d level & & 1 & 4 & 1 & 3 & & 5 & 1 & 12 \\
\hline \multicolumn{10}{|l|}{ Environmental factors } \\
\hline 2nd level & 13 & 9 & & & 1 & & 7 & & \\
\hline 3d level & 3 & & & & & & & & \\
\hline \multicolumn{10}{|l|}{ Not classified } \\
\hline Nc-health condition & 6 & & & 2 & & & & 2 & \\
\hline Nc-Quality of life & & & 1 & 2 & & & & 13 & 5 \\
\hline Nd-general health & & & 1 & & & & & 2 & 6 \\
\hline Nd-physical health & & & 3 & & & & & 1 & 2 \\
\hline Nd-mental health & & & & & & & & & 2 \\
\hline Nd-disability & 2 & & & & & & & & \\
\hline Personal factors & 1 & & & & & & & 1 & 2 \\
\hline
\end{tabular}

Desc, descriptive; Dep, dependency; Appr, appraisal; Int, intensity; NC, not covered; ND, not definable 
Structures, 50 - under Activities and Participation, and 18 - Environmental Factors. Detailed comparison of the content between assessment tools are shown in Tables 4, 5, and 6 for components of Body Functions and Structures, Activities and Participation and Environmental Factors, respectively. No appropriate ICF category was found for 54 items following the ICF linking guidelines.

Evaluating all 9 assessment tools, the most related ICF categories in the body function domain were b140 attention functions $(\mathrm{n}=5)$, b144 memory functions $(\mathrm{n}=4)$, b156 perceptual functions $(n=4)$, b280 sensation of pain $(\mathrm{n}=4)$ and b730 muscle power functions $(\mathrm{n}=4)$. FACT$\mathrm{BR}, \mathrm{BN}-20$ and MMSE contained most concepts related to the Body functions and Structures. Five out of nine analyzed assessment tools included concepts on d450 walking and $\mathrm{d} 540$ dressing. The $\mathrm{BN}-20$ questionnaire contained meaningful concepts that could be linked to 23 s level categories of the Activities and Participation, covering all domains of this component. FIM was linked to 21 categories that did not cover chapters of Major life areas and Community, social and civic life.

\section{Psychometric properties}

For psychometric properties that are specific for brain tumor diagnosis, search in PubMed yielded 578 results for KPS, 18 for MMSE, 55 for EORTC QLQ-C30, 6 for EORTC QLQ-BN20, 5 for FIM, 36 for TMT, 14 for BI, 21 for FACT-Br, and 4 for SF-36. Headline screening resulted in identifying 1 study for EORTC QLQ-C30, 4 studies for EORTC QLQ-BN20, 3 studies for FACT- Br, and 1 study for SF-36. A search strategy for various diagnoses was implemented for the remaining assessment instruments as well as SF-36 and EORTC QLQ-C30 due to the previous search strategy yielding only 1 result. As a result, for further analysis, 4 articles for KPS, 5 for MMSE, 1+5 for EORTC QLQ-C30, 4 for EORTC QLQBN20, 10 for FIM, 2 for TMT, 7 for BI 3 for FACT-Br, and 8 for SF-36 were included in this review. The psychometric properties for assessment instruments EORTC QLQC30, EORTC QLQ-BN20, FACT-Br, and SF-36, that are specific to brain tumor diagnosis are summarized in Table 7. The psychometric properties analyzed in mixed diagnosis studies for EORTC QLQ-C30, MMSE, SF-36, BI, FIM, KPS, TMT are summarized below, see Table 8 .

\section{Discussion}

This study identified nine rehabilitation assessment instruments that have most commonly been referred to in the literature for adults with brain tumors, that cover all components of the ICF, and have good psychometric properties. As far as the authors are aware, this is the only scoping review of assessment instruments used for adults with brain tumors. However, this scoping review did not identify one unique assessment instrument for the target group. This patient group is specific in a way that there is no unifying patient-specific clinical set of symptoms and their symptoms depend on various other factors [14].

Five of these tools are used for objective assessment: KPS, MMSE, FIM, TMT, BI, four are self-assessment tools: FACT-Br, SF-36, EORTC QLQ-C30, EORTC QLQ$\mathrm{BN20}$. One of these tools (KPS) is used to assess physical activity, two (MMSE; TMT) are cognitive function assessment tools, FACT-Br, SF-36, EORTC QLQ-C30, EORTC QLQ-BN20 measures the quality of life, and both, FIM and BI are used to assess disability.

The most frequently used assessment instrument is the Karnofsky Performance Scale as it is used as a criterion for the selection of participants by measuring their level of physical activity [15]. This assessment tool is developed for a general assessment of oncological patients [16] and reflects the overall ability to perform usual daily activities (component of Activities and Participation of the ICF) in the context of help needed from other people (Environmental Factors).

Four of these instruments are used to evaluate patients with brain tumors: EORTC QLQ-C30; EORTC QLQBN20; FACT-Br, SF-36; they are all linked to the quality of life. Moreover, the EORTC team recommends that EORTC QLQ-C30 and EORTC QLQ-BN20 tools be used together [17]. These two tools cover both functioning components of the ICF and from the perspective of content, complement each other. EORTC QLQ-C30 contains more specific questions on problems specific to patients with brain tumors $[18,19]$. The EORTC QLQ-C30 and the EORTC QLQ-BN20 provide comprehensive information about the patient's quality of life, but this is often overlooked in studies identified in this scoping review. The FACT-Br questionnaire has been used less and it has as good properties in terms of intra-rater reliability and structural validity as other two specific quality of life measurements, contains problems that have not been included in any of the previous tools, and can clearly be important for this population, such as handling stress or driving a car. It also considers important Environmental factors, such as help and attitudes of family members and friends, as well as health professionals. Some important concepts also overlap with the SF-36 that have developed as a multipurpose tool that is used for assessment of functional health and well-being [20] and has also been widely used for patient-reported outcomes in populations with different diagnoses [21]. Therefore, this could be a good choice to use the SF-36, if the comparison between populations is needed.

Between the most used assessment tools, the FIM and BI have been listed. These instruments are non-specific to diagnosis, and both have been widely used in different 


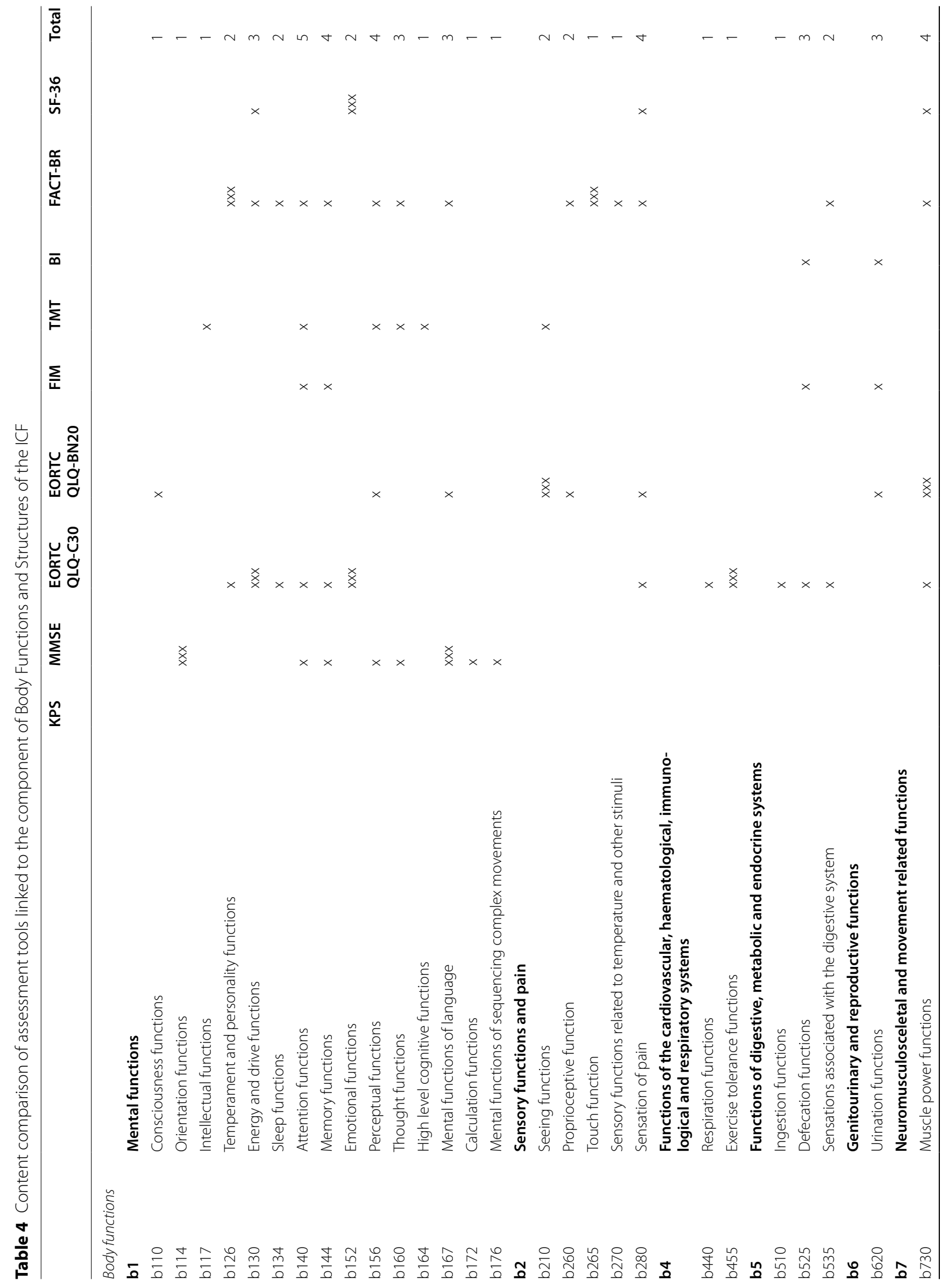




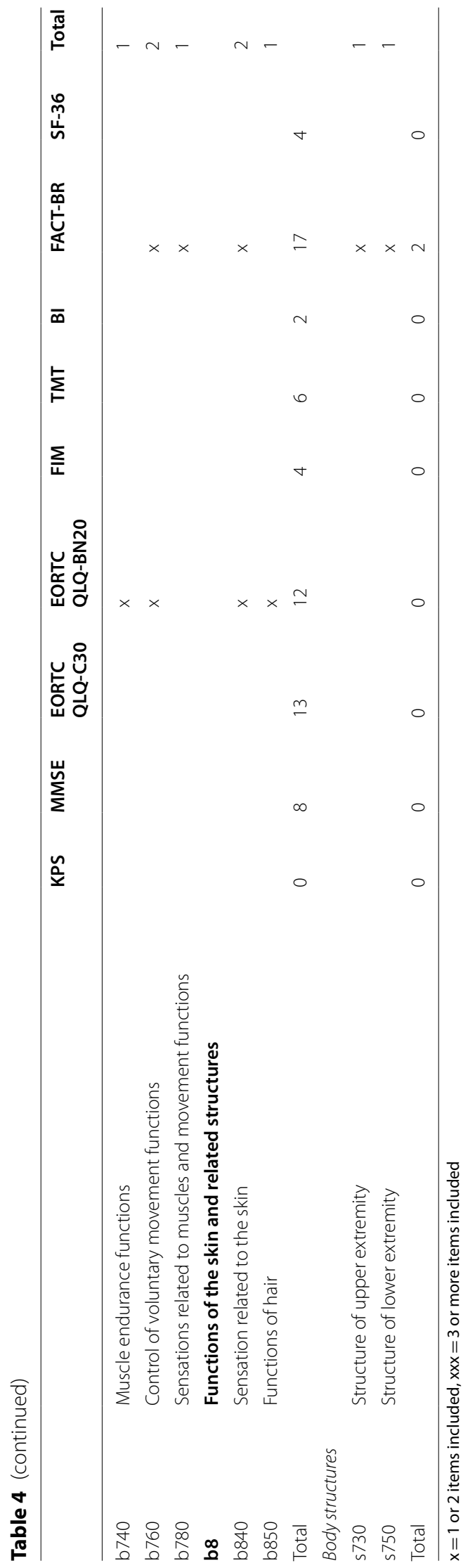


Table 5 Content comparison of assessment tools linked to the component of Activities and Participation of the ICF

\begin{tabular}{|c|c|c|c|c|c|c|c|c|c|c|c|}
\hline & & KPS & MMSE & $\begin{array}{l}\text { EORTC } \\
\text { QLQ- } \\
\text { C30 }\end{array}$ & $\begin{array}{l}\text { EORTC } \\
\text { QLQ-BN20 }\end{array}$ & FIM & TMT & BI & FACT-BR & SF-36 & Total \\
\hline d1 & Learning and applying knowledge & & & & & & & & & $x x x$ & 1 \\
\hline d110 & Watching & & & $x$ & & & $x$ & & & & 2 \\
\hline d115 & Listening & & & & & & $x$ & & & & 1 \\
\hline d160 & Focusing attention & & & & & & $x$ & & & & 1 \\
\hline d163 & Thinking & & & & & & $x$ & & & & 1 \\
\hline d166 & Reading & & & $x$ & $x$ & & $x$ & & $x$ & & 4 \\
\hline d175 & Solvingproblems & & & & & $x$ & & & $x$ & & 2 \\
\hline d177 & Making decisions & & & & & & & & $x$ & & 1 \\
\hline d2 & General tasks and demands & & & & & & & & & & \\
\hline$d 220$ & Undertaking multiple tasks & & & & & & $x$ & & & & 1 \\
\hline$d 230$ & Carrying out daily routine & & & $x$ & & & & & & $x$ & 2 \\
\hline$d 240$ & Handling stress and other psychological demands & & & & & & & & $x$ & & 1 \\
\hline d3 & Communication & & & & & & & & $x$ & & 1 \\
\hline d310 & Communicating with receiving spoken messages & & & & & $x$ & & & & & 1 \\
\hline d315 & Communicating with receiving nonverbal messages & & & & & $x$ & $x$ & & & & 2 \\
\hline d320 & $\begin{array}{l}\text { Communicating with_receiving_formal sign } \\
\text { language messages }\end{array}$ & & & & & $x$ & & & & & 1 \\
\hline d325 & Communicating with—receiving—written messages & & & & & $x$ & & & & & 1 \\
\hline d330 & Speaking & & & & $x$ & $x$ & & & $x$ & & 3 \\
\hline d335 & Producing nonverbal messages & & $x$ & & & $x$ & & & & & 2 \\
\hline d340 & Lifting and carrying objects & & & $x$ & & $x$ & & & & & 2 \\
\hline d345 & Writing messages & & & & & $x$ & & & $x$ & & 2 \\
\hline d350 & Conversation & & & & $x$ & & & & & & 1 \\
\hline d4 & Mobility & & & $x$ & & & & & & & 1 \\
\hline$d 410$ & Changing basic body position & & & & & & & $x$ & & $x$ & 2 \\
\hline$d 415$ & Maintaining a body position & & & $x$ & $x$ & & & & $x$ & & 3 \\
\hline$d 420$ & Transferring oneself & & & & & $x x x$ & & $x$ & & & 2 \\
\hline$d 430$ & Lifting and carrying objects & & & & & & & & & $x x x$ & 1 \\
\hline$d 440$ & Fine hand use & & & & & & $x$ & & & & 1 \\
\hline$d 455$ & Hand and arm use & & & & & & $x$ & & & & 1 \\
\hline$d 450$ & Walking & & & $x$ & $x$ & $x$ & & $x x x$ & & $x x x$ & 5 \\
\hline d455 & Moving around & & & & & $x$ & & $x x x$ & & $x x x$ & 3 \\
\hline$d 460$ & Moving around in different locations & & & $x$ & & & & & & & 1 \\
\hline$d 465$ & Moving around using equipment & & & & & $x$ & & $x$ & & & 2 \\
\hline$d 475$ & Driving & & & & & & & & $x$ & & 1 \\
\hline d5 & Self-care & $x x x$ & & & & & & & $x$ & & 2 \\
\hline d510 & Washing oneself & & & $x$ & & $x$ & & $x$ & & $x$ & 4 \\
\hline d520 & Caring for body parts & & & & & $x$ & & $x x x$ & $x$ & & 3 \\
\hline d530 & Toileting & & & $x$ & & $x$ & & $x$ & & & 3 \\
\hline d540 & Dressing & & & $x$ & & $x$ & & $x$ & $x$ & $x$ & 5 \\
\hline d550 & Eating & & & $x$ & & $x$ & & $x$ & $x$ & & 4 \\
\hline d560 & Drinking & & & & & & & & $x$ & & 1 \\
\hline d598 & Self-care & & & & & & & & & & 0 \\
\hline d6 & Domestic life & & & $x$ & & & & & & $x$ & 2 \\
\hline$d 620$ & Acquisition of goods and services & & & & & $x$ & & & & & 1 \\
\hline d7 & Interpersonal interactions and relationships & & & $x$ & & & & & & $x$ & 2 \\
\hline$d 710$ & Basic interpersonal interactions & & & & & $x$ & & & & & 1 \\
\hline$d 720$ & Complex interpersonal interactions & & & & & $x$ & & & & & 1 \\
\hline
\end{tabular}


Table 5 (continued)

\begin{tabular}{|c|c|c|c|c|c|c|c|c|c|c|c|}
\hline & & KPS & MMSE & $\begin{array}{l}\text { EORTC } \\
\text { QLQ- } \\
\text { C30 }\end{array}$ & $\begin{array}{l}\text { EORTC } \\
\text { QLQ-BN20 }\end{array}$ & FIM & TMT & BI & FACT-BR & SF-36 & Total \\
\hline$d 750$ & Informal social relationships & & & & & & & & & $x x x$ & 1 \\
\hline$d 760$ & Family relationships & & & $x$ & $x$ & & & & $x$ & $x$ & 4 \\
\hline$d 770$ & Intimate relationships & & & & & & & & $x$ & & 1 \\
\hline d8 & Major life areas & & & $x$ & & & & & & $x$ & 2 \\
\hline d840 & Apprenticeship (work preparation) & $x x x$ & & $x$ & & & & & & & 2 \\
\hline $\mathrm{d} 845$ & Acquiring, keeping and terminating a job & $x x x$ & & $x$ & & & & & & & 2 \\
\hline d850 & Remunerative employment & $x x x$ & & $x$ & & & & & $x$ & $x$ & 4 \\
\hline d855 & Non-remunerative employment & $x x x$ & & $x$ & & & & & & & 2 \\
\hline $\mathrm{d} 870$ & Economic self-sufficiency & & & $x$ & & & & & & & 1 \\
\hline d9 & Community, social and civic life & & & $x$ & & & & & & $x$ & 2 \\
\hline d910 & Community life & & & & & & & & $x$ & & 1 \\
\hline d920 & Recreation and leisure & & & $x$ & & & & & $x$ & $x x x$ & 3 \\
\hline Total & & 6 & 1 & 23 & 6 & 21 & 9 & 10 & 19 & 16 & \\
\hline
\end{tabular}

$\mathrm{x}=1$ or 2 items included, $\mathrm{xx}=3$ or more items included

rehabilitation populations [22-24]. Both scales, the FIM and the BI, are performance-based assessment tools and both analyze the level of independence in the most important activities of daily living. Their psychometric properties have been profoundly analyzed, and the ceiling effect for the BI can be observed when compared to the FIM [25]. However, the psychometric properties of the objective assessment instruments specific for the patient group have not been proven; therefore, their psychometric properties were demonstrated in patients suffering from stroke, traumatic brain injury (TBI), or similar neurological conditions. Interestingly, two neuropsychological assessment tools (the MMSE and the TMT) are mentioned among the most frequently used for persons with brain tumors. It can be explained by the fact that cognitive impairments are a common symptom in patients with brain tumors [4]. Both instruments focus mostly on the cognitive functions of the component of the Body Functions and Structures of the ICF and both are performance-based. However, the psychometric properties of the MMSE have been better documented.

The International Classification of Functioning, Disability and Health (ICF) provides the user with a broad spectrum of health outcomes, including physical and cognitive functioning. By linking available assessment tools to this concept, it is possible to analyze the content of the available instruments and choose the appropriate one for the problem that is being measured and, consequently, treated $[11,12]$. Using the ICF framework, it was possible to link most elements identified in the assessment instruments to certain categories. Some elements could not be linked since they covered topics such as quality of life, personal factors, or certain elements not defined in the ICF. Body Function categories were dominated by MMSE, TMT, EORTC QLQ-BN20, EORTC QLQC30, activity and participation categories-FIM, BI, KPS, SF-36 but FACT-Br viewed these two domains equally. Environmental factors were assessed by EORTC QLQ30, FACT-Br, FIM, BI, and KPS. Given that the clinical picture of brain tumor patients is similar to that of other neurological conditions, such as stroke [6] or TBI [7], the ICF Core Sets were reviewed for stroke and TBI [26], and their categories were compared to categories identified in this scoping review. Comprehensive core sets for stroke listed 13 categories in body functions and structures, 14 in activities and participation, and 23 categories in environmental factors that were not identified in assessment instruments analyzed in this study. Comprehensive core sets for TBI listed 10 categories in body functions domain, 22 in activities and participation, and 28 categories in environmental factor domain that were not identified in any of 9 assessment instruments analyzed within this study. This can be explained by the fact that the most frequently used assessment instruments do not cover all the possible impairments for people with brain tumors.

Given that the ICF Core Sets for stroke and TBI were compared to categories identified in this review and they proved to be overall covering similar areas it can be concluded that all 9 assessment tools identified in this study can be appropriate and specific assessment instruments for patients with brain tumors, as they have been proven valid, reliable, and responsive to a variety of neurological conditions. Further research is recommended to assess 


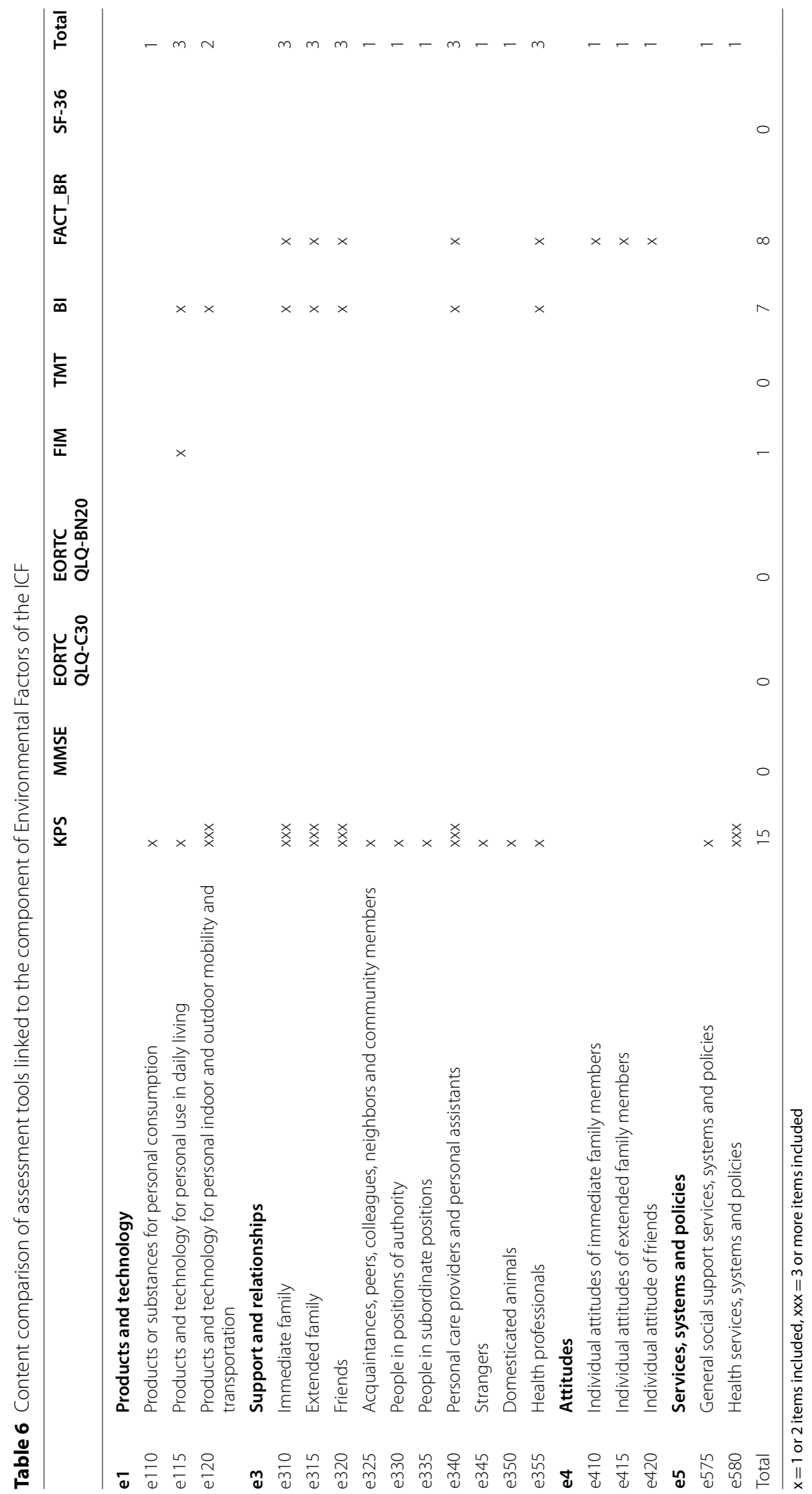




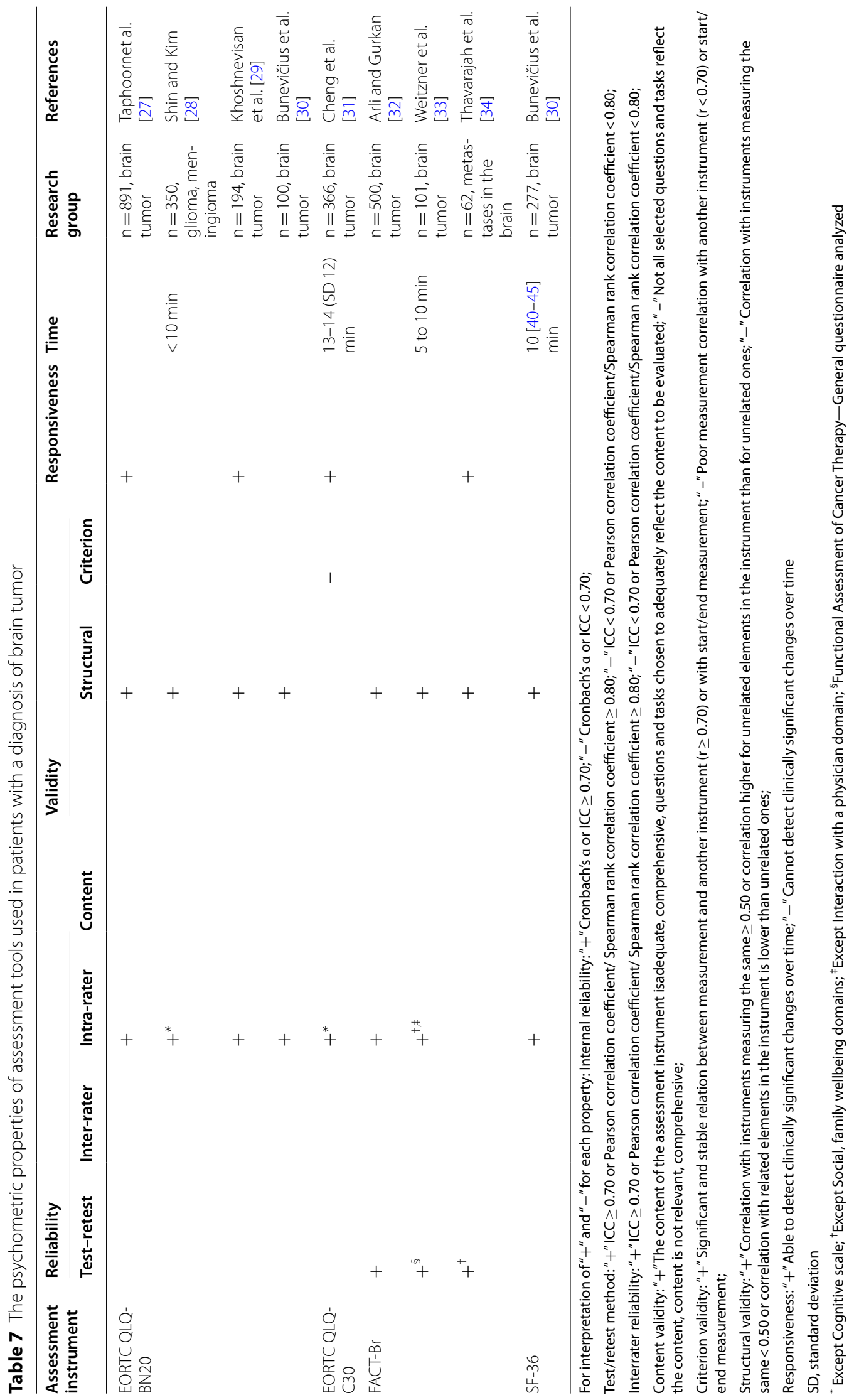




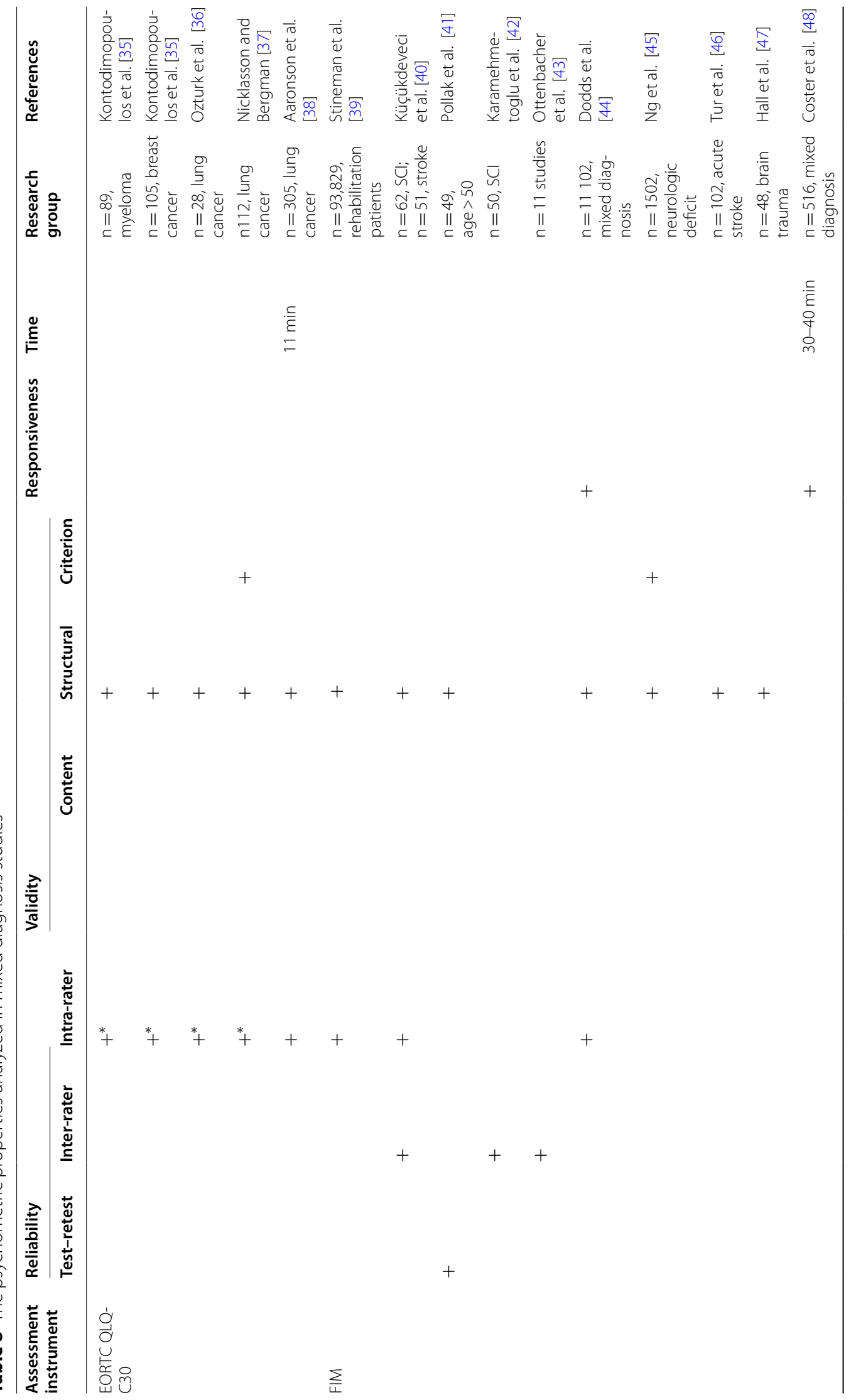




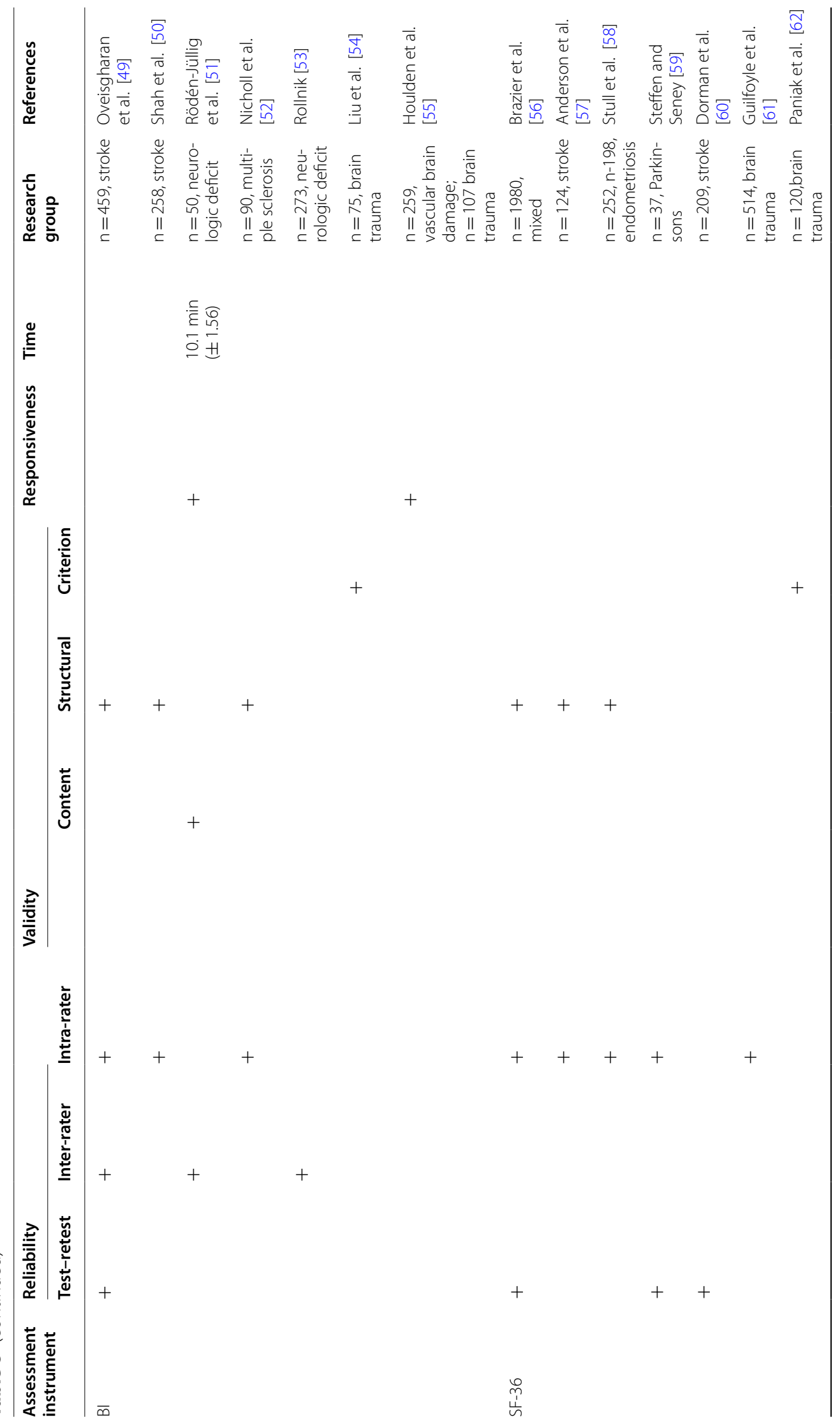




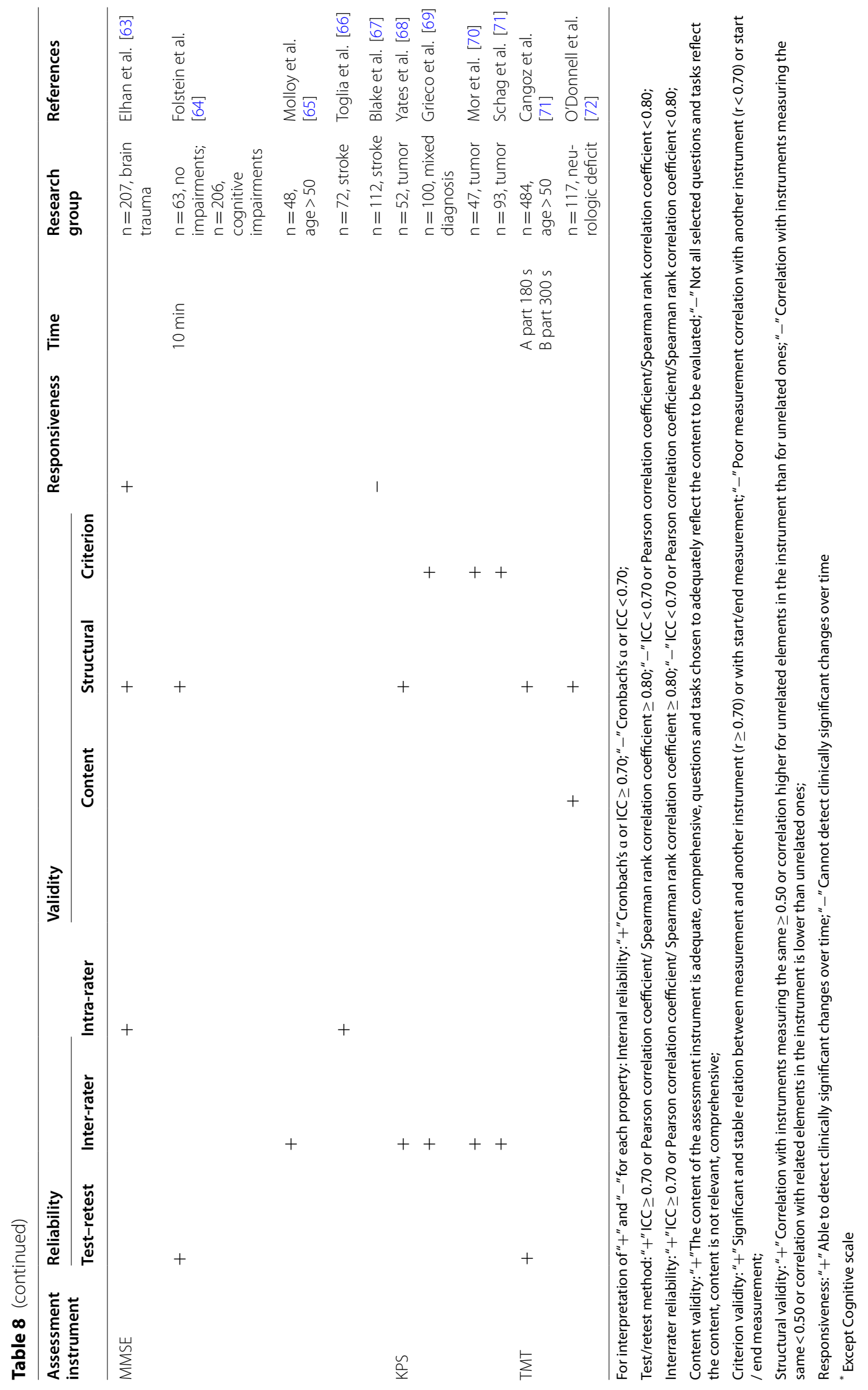


reliability, validity, and responsiveness of assessment instruments specifically for brain tumor patient groups.

Overall, the current study has a few limitations. First, the quality of the studies included in the scoping review was not assessed, as the purpose of the scoping review was to identify the most frequently used assessment instruments. Second, the authors included only nine out of 86 assessment tools for further analysis, which were used in more than $9 \%$ of the study articles included in the study. That runs the risk that this analysis of assessment instruments does not use some of the more recently developed assessment tools, which may be better suited for the specific patient group but are not used frequently enough in research articles to be included in the analysis.

\section{Conclusions}

Between the nine most frequently used assessment instruments in clinical studies, one was a generic tool for an overall description of activity level for patients with diagnosis of cancer, three were diagnosis-specific selfassessment tools, one was a multipurpose tool for assessment of functionality and health status, two were widely used tools in rehabilitation for assessment of activities of daily living, and two were neurocognitive tests. These tools cover all components of the International Classification of Functioning, Disability and Health and have proven to have good psychometric properties; however, the assessment tools that are not diagnosis-specific, still must be validated for the brain tumor population.

Since the content and administration vary, the choice of the tool used for assessment of patients with brain tumor depends on the clinical question posed, as well as the aim of the use of this tool.

\section{Supplementary Information}

The online version contains supplementary material available at https://doi. org/10.1186/s12955-021-01863-0.

Additional file 1. All identified assessment tools, frequency and references.

\section{Acknowledgements}

Not applicable.

\section{Authors' contributions}

LG developed the design and study protocol, were involved in data analysis and interpretation, AP were involved in the data analysis, interpretation of results and preparing the manuscript, SC were involved in the data analysis, GB were involved in the development of study protocol, data interpretation and preparing the manuscript. All authors read and approved the final manuscript.

\section{Funding}

This study was supported by Riga Stradiņš University internal grant.
Availability of data and materials

The datasets used and/or analysed during the current study are available from the corresponding author on reasonable request.

\section{Declarations}

Ethics approval and consent to participate.

Not applicable.

\section{Consent for publication}

Not applicable.

\section{Competing interests}

The authors declare that they have no competing interests.

\section{Author details}

${ }^{1}$ Riga Stradinš̌ University, Riga, Latvia. ${ }^{2}$ Riga East University Hospital, Riga, Latvia. ${ }^{3}$ Department of Rehabilitation, Riga Stradiņ̧̌ University, Anniņmuižas Boulevard 26a, Riga 1067, Latvia.

Received: 10 September 2020 Accepted: 15 September 2021

Published online: 09 October 2021

\section{References}

1. Statistikas dati par onkoloǵiskiem pacientiem, 2010-2017. The Centre for Disease Prevention and Control of Latvia; 2020.

2. Koshy M, Villano JL, Dolecek TA, Howard A, Mahmood U, Chmura SJ, et al. Improved survival time trends for glioblastoma using the SEER 17 population-based registries. J Neurooncol. 2012;107(1):207-12.

3. Kong X, Ma W, Li Y, Wang Y, Guan J, Gao J, et al. Does tenascin have clinical implications in pathological grade of glioma patients?: A systematic meta-analysis. Medicine (Baltimore). 2015;94(32):e1330.

4. Mukand JA, Blackinton DD, Crincoli MG, Lee JJ, Santos BB. Incidence of neurologic deficits and rehabilitation of patients with brain tumors. Am J Phys Med Rehabil. 2001;80(5):346-50.

5. Bartolo M, Zucchella C, Pace A, Lanzetta G, Vecchione C, Bartolo M, et al. Early rehabilitation after surgery improves functional outcome in inpatients with brain tumours. J Neurooncol. 2012;107(3):537-44.

6. Huang ME, Cifu DX, Keyser-Marcus L. Functional outcome after brain tumor and acute stroke: a comparative analysis. Arch Phys Med Rehabil. 1998;79(11):1386-90.

7. Huang ME, Cifu DX, Keyser-Marcus L. Functional outcomes in patients with brain tumor after inpatient rehabilitation: comparison with traumatic brain injury. Am J Phys Med Rehabil. 2000;79(4):327-35.

8. Khan FAB, Rajapaksa I, Ng L. Outcomes of social support programs in brain cancer survivors in an Australian community cohort: a prospective study. J Cancer Res Therapy. 2013;1:24-33.

9. Salomon JAMC, Chatterji S, Sadana R, Üstün TB, Murray JL. Quantifying individual levels of health: definitions, concepts, and measurement issues. In: Murray CJLED, editor. Health systems performance assessment debates, methods and empiricism. Geneva: World Health Organization; 2003. p. 301-18.

10. Peters MDJGC, McInerney P, Munn Z, Tricco AC, Khalil H. Chapter 11: Scoping reviews (2020 version). In: Romataris E, Munn Z, editors. JBI manual for evidence synthesis; 2020.

11. Cieza A, Fayed N, Bickenbach J, Prodinger B. Refinements of the ICF Linking Rules to strengthen their potential for establishing comparability of health information. Disabil Rehabil. 2019;41(5):574-83.

12. Cieza A, Geyh S, Chatterji S, Kostanjsek N, Ustun B, Stucki G. ICF linking rules: an update based on lessons learned. J Rehabil Med. 2005;37(4):212-8.

13. Terwee CB, Jansma EP, Riphagen II, de Vet HC. Development of a methodological PubMed search filter for finding studies on measurement properties of measurement instruments. Qual Life Res. 2009;18(8):1115-23.

14. M G. Hand book of neurosurgery. 8th edn. New York: Thieme; 2016.

15. Peus D, Newcomb N, Hofer S. Appraisal of the Karnofsky Performance Status and proposal of a simple algorithmic system for its evaluation. BMC Med Inform Decis Mak. 2013;13:72. 
16. Terret C, Albrand G, Moncenix G, Droz JP. Karnofsky Performance Scale (KPS) or Physical Performance Test (PPT)? That is the question. Crit Rev Oncol Hematol. 2011;77(2):142-7.

17. Fayers PAN, Bjordal K, Groenvold M, Curran D, Bottomley A. EORTC QLQC30 Scoring Manual. 3d ed. Brussels: European Organisation for Research and Treatment of Cancer; 2001.

18. Flechl B, Ackerl M, Sax C, Oberndorfer S, Calabek B, Sizoo E, et al. The caregivers' perspective on the end-of-life phase of glioblastoma patients. J Neurooncol. 2013;112(3):403-11.

19. Arber A, Faithfull S, Plaskota M, Lucas C, de Vries K. A study of patients with a primary malignant brain tumour and their carers: symptoms and access to services. Int J Palliat Nurs. 2010;16(1):24-30.

20. Contopoulos-Ioannidis DG, Karvouni A, Kouri I, loannidis JP. Reporting and interpretation of SF-36 outcomes in randomised trials: Systematic review. BMJ. 2009;338:a3006

21. Scoggins JF, Patrick DL. The use of patient-reported outcomes instruments in registered clinical trials: evidence from ClinicalTrials.gov. Contemp Clin Trials. 2009:30(4):289-92.

22. Hopman-Rock M, van Hirtum $H$, de Vreede P, Freiberger E. Activities of daily living in older community-dwelling persons: a systematic review of psychometric properties of instruments. Aging Clin Exp Res. 2019;31(7):917-25.

23. Laxe S, Tschiesner U, Zasler N, Lopez-Blazquez R, Tormos JM, Bernabeu M. What domains of the International Classification of Functioning, Disability and Health are covered by the most commonly used measurement instruments in traumatic brain injury research? Clin Neurol Neurosurg 2012:114(6):645-50

24. Sivan M, O'Connor RJ, Makower S, Levesley M, Bhakta B. Systematic review of outcome measures used in the evaluation of robot-assisted upper limb exercise in stroke. J Rehabil Med. 2011;43(3):181-9.

25. Prodinger B, O'Connor RJ, Stucki G, Tennant A. Establishing score equivalence of the Functional Independence Measure motor scale and the Barthel Index, utilising the International Classification of Functioning, Disability and Health and Rasch measurement theory. J Rehabil Med. 2017:49(5):416-22.

26. ICF Research Branch: ICF Research Branch, a cooperation partner within the WHO Collaborating Centre for the Family of International Classifications in Germany (at DIMDI); 2017. https://www.icf-research-branch.org/ icf-core-sets

27. Taphoorn MJ, Claassens L, Aaronson NK, Coens C, Mauer M, Osoba D, et al. An international validation study of the EORTC brain cancer module (EORTC QLQ-BN20) for assessing health-related quality of life and symptoms in brain cancer patients. Eur J Cancer. 2010;46(6):1033-40.

28. Shin YS, Kim JH. Validation of the Korean version of the European Organization for Research and Treatment of Cancer brain cancer module (EORTC QLQ-BN20) in patients with brain tumors. Health Qual Life Outcomes. 2013;11:145.

29. Khoshnevisan A, Yekaninejad MS, Ardakani SK, Pakpour AH, Mardani A, Aaronson NK. Translation and validation of the EORTC brain cancer module (EORTC QLQ-BN20) for use in Iran. Health Qual Life Outcomes. 2012;10:54.

30. Bunevicius A, Tamasauskas S, Tamasauskas A, Deltuva V. Evaluation of health-related quality of life in Lithuanian brain tumor patients using the EORTC brain cancer module. Medicina (Kaunas). 2012;48(11):588-94.

31. Cheng JX, Liu BL, Zhang X, Zhang YQ, Lin W, Wang R, et al. The validation of the standard Chinese version of the European Organization for Research and Treatment of Cancer Quality of Life Core Questionnaire 30 (EORTC QLQ-C30) in pre-operative patients with brain tumor in China. BMC Med Res Methodol. 2011;11:56

32. Arli SK, Gurkan A. Validity and reliability of Turkish version of the functional assessment of cancer therapy-brain questionnaire. Cancer Nurs. 2017:40(3):224-9.

33. Weitzner MA, Meyers CA, Gelke CK, Byrne KS, Cella DF, Levin VA. The Functional Assessment of Cancer Therapy (FACT) scale. Development of a brain subscale and revalidation of the general version (FACT-G) in patients with primary brain tumors. Cancer. 1995;75(5):1151-61.

34. Thavarajah N, Bedard G, Zhang L, Cella D, Beaumont JL, Tsao M, et al. Psychometric validation of the functional assessment of cancer therapybrain (FACT-Br) for assessing quality of life in patients with brain metastases. Support Care Cancer. 2014;22(4):1017-28.
35. Kontodimopoulos N, Ntinoulis K, Niakas D. Validity of the Greek EORTC QLQ-C30 and QLQ-BR23 for measuring health-related quality of life in breast cancer patients. Eur J Cancer Care (Engl). 2011;20(3):354-61.

36. Ozturk A, Sarihan S, Ercan I, Karadag M. Evaluating quality of life and pulmonary function of long-term survivors of non-small cell lung cancer treated with radical or postoperative radiotherapy. Am J Clin Oncol. 2009:32(1):65-72

37. Nicklasson M, Bergman B. Validity, reliability and clinical relevance of EORTC QLQ-C30 and LC13 in patients with chest malignancies in a palliative setting. Qual Life Res. 2007;16(6):1019-28.

38. Aaronson NK, Ahmedzai S, Bergman B, Bullinger M, Cull A, Duez NJ, et al. The European Organization for Research and Treatment of Cancer QLQC30: a quality-of-life instrument for use in international clinical trials in oncology. J Natl Cancer Inst. 1993;85(5):365-76.

39. Stineman MG, Shea JA, Jette A, Tassoni CJ, Ottenbacher KJ, Fiedler R, et al. The Functional Independence Measure: tests of scaling assumptions, structure, and reliability across 20 diverse impairment categories. Arch Phys Med Rehabil. 1996;77(11):1101-8.

40. Kucukdeveci AA, Yavuzer G, Elhan AH, Sonel B, Tennant A. Adaptation of the functional independence measure for use in Turkey. Clin Rehabil. 2001;15(3):311-9.

41. Pollak N, Rheault W, Stoecker JL. Reliability and validity of the FIM for persons aged 80 years and above from a multilevel continuing care retirement community. Arch Phys Med Rehabil. 1996;77(10):1056-61.

42. Karamehmetoglu SS, Karacan I, Elbasi N, Demirel G, Koyuncu H, Dosoglu $M$. The functional independence measure in spinal cord injured patients: comparison of questioning with observational rating. Spinal Cord. 1997;35(1):22-5.

43. Ottenbacher KJ, Hsu Y, Granger CV, Fiedler RC. The reliability of the functional independence measure: a quantitative review. Arch Phys Med Rehabil. 1996:77(12):1226-32.

44. Dodds TA, Martin DP, Stolov WC, Deyo RA. A validation of the functional independence measurement and its performance among rehabilitation inpatients. Arch Phys Med Rehabil. 1993;74(5):531-6.

45. Ng YS, Jung $H$, Tay SS, Bok CW, Chiong Y, Lim PA. Results from a prospective acute inpatient rehabilitation database: clinical characteristics and functional outcomes using the Functional Independence Measure. Ann Acad Med Singapore. 2007:36(1):3-10.

46. Tur BS, Gursel YK, Yavuzer G, Kucukdeveci A, Arasil T. Rehabilitation outcome of Turkish stroke patients: in a team approach setting. Int J Rehabil Res. 2003;26(4):271-7

47. Hall KM, Bushnik T, Lakisic-Kazazic B, Wright J, Cantagallo A. Assessing traumatic brain injury outcome measures for long-term follow-up of community-based individuals. Arch Phys Med Rehabil. 2001;82(3):367-74.

48. Coster WJ, Haley SM, Jette AM. Measuring patient-reported outcomes after discharge from inpatient rehabilitation settings. J Rehabil Med. 2006;38(4):237-42

49. Oveisgharan S, Shirani S, Ghorbani A, Soltanzade A, Baghaei A, Hosseini $S$, et al. Barthel index in a Middle-East country: translation, validity and reliability. Cerebrovasc Dis. 2006;22(5-6):350-4.

50. Shah S, Vanclay F, Cooper B. Improving the sensitivity of the Barthel Index for stroke rehabilitation. J Clin Epidemiol. 1989;42(8):703-9.

51. Roden-Jullig A, Britton M, Gustafsson C, Fugl-Meyer A. Validation of four scales for the acute stage of stroke. J Intern Med. 1994;236(2):125-36.

52. Nicholl L, Hobart J, Dunwoody L, Cramp F, Lowe-Strong A. Measuring disability in multiple sclerosis: is the Community Dependency Index an improvement on the Barthel Index? Mult Scler. 2004;10(4):447-50.

53. Rollnik JD. The Early Rehabilitation Barthel Index (ERBI). Rehabilitation (Stuttg). 2011;50(6):408-11.

54. Liu C, McNeil JE, Greenwood R. Rehabilitation outcomes after brain injury: disability measures or goal achievement? Clin Rehabil. 2004;18(4):398-404

55. Houlden H, Edwards M, McNeil J, Greenwood R. Use of the Barthel Index and the Functional Independence Measure during early inpatient rehabilitation after single incident brain injury. Clin Rehabil. 2006;20(2):153-9.

56. Brazier JE, Harper R, Jones NM, O'Cathain A, Thomas KJ, Usherwood T, et al. Validating the SF-36 health survey questionnaire: new outcome measure for primary care. BMJ. 1992;305(6846):160-4.

57. Anderson C, Laubscher S, Burns R. Validation of the Short Form 36 (SF-36) health survey questionnaire among stroke patients. Stroke. $1996 \cdot 27(10) \cdot 1812-6$ 
58. Stull DE, Wasiak R, Kreif N, Raluy M, Colligs A, Seitz C, et al. Validation of the SF-36 in patients with endometriosis. Qual Life Res. 2014;23(1):103-17.

59. Steffen T, Seney M. Test-retest reliability and minimal detectable change on balance and ambulation tests, the 36-item short-form health survey, and the unified Parkinson disease rating scale in people with parkinsonism. Phys Ther. 2008;88(6):733-46.

60. Dorman P, Slattery J, Farrell B, Dennis M, Sandercock P. Qualitative comparison of the reliability of health status assessments with the EuroQol and SF-36 questionnaires after stroke. United Kingdom Collaborators in the International Stroke Trial. Stroke. 1998;29(1):63-8.

61. Guilfoyle MR, Seeley HM, Corteen E, Harkin C, Richards H, Menon DK, et al. Assessing quality of life after traumatic brain injury: examination of the short form 36 health survey. J Neurotrauma. 2010;27(12):2173-81.

62. Paniak C, Phillips K, Toller-Lobe G, Durand A, Nagy J. Sensitivity of three recent questionnaires to mild traumatic brain injury-related effects. J Head Trauma Rehabil. 1999;14(3):211-9.

63. Elhan AH, Kutlay S, Kucukdeveci AA, Cotuk C, Ozturk G, Tesio L, et al. Psychometric properties of the Mini-Mental State Examination in patients with acquired brain injury in Turkey. J Rehabil Med. 2005;37(5):306-11.

64. Folstein MF, Folstein SE, McHugh PR. "Mini-mental state". A practical method for grading the cognitive state of patients for the clinician. J Psychiatr Res. 1975;12(3):189-98.

65. Molloy DW, Standish TI. A guide to the standardized Mini-Mental State Examination. Int Psychogeriatr. 1997;9(Suppl 1):87-94.

66. Toglia J, Fitzgerald KA, O'Dell MW, Mastrogiovanni AR, Lin CD. The MiniMental State Examination and Montreal Cognitive Assessment in persons with mild subacute stroke: relationship to functional outcome. Arch Phys Med Rehabil. 2011;92(5):792-8.

67. Blake H, McKinney M, Treece K, Lee E, Lincoln NB. An evaluation of screening measures for cognitive impairment after stroke. Age Ageing. 2002;31(6):451-6.

68. Yates JW, Chalmer B, McKegney FP. Evaluation of patients with advanced cancer using the Karnofsky performance status. Cancer. 1980;45(8):2220-4.

69. Grieco A, Long CJ. Investigation of the Karnofsky Performance Status as a measure of quality of life. Health Psychol. 1984;3(2):129-42.

70. Mor V, Laliberte L, Morris JN, Wiemann M. The Karnofsky Performance Status Scale. An examination of its reliability and validity in a research setting. Cancer. 1984;53(9):2002-7.

71. Schag CC, Heinrich RL, Ganz PA. Karnofsky performance status revisited: reliability, validity, and guidelines. J Clin Oncol. 1984;2(3):187-93.

72. O'Donnell JP, Macgregor LA, Dabrowski JJ, Oestreicher JM, Romero JJ. Construct validity of neuropsychological tests of conceptual and attentional abilities. J Clin Psychol. 1994;50(4):596-600.

\section{Publisher's Note}

Springer Nature remains neutral with regard to jurisdictional claims in published maps and institutional affiliations.
Ready to submit your research? Choose BMC and benefit from:

- fast, convenient online submission

- thorough peer review by experienced researchers in your field

- rapid publication on acceptance

- support for research data, including large and complex data types

- gold Open Access which fosters wider collaboration and increased citations

- maximum visibility for your research: over 100M website views per year

At BMC, research is always in progress.

Learn more biomedcentral.com/submissions 